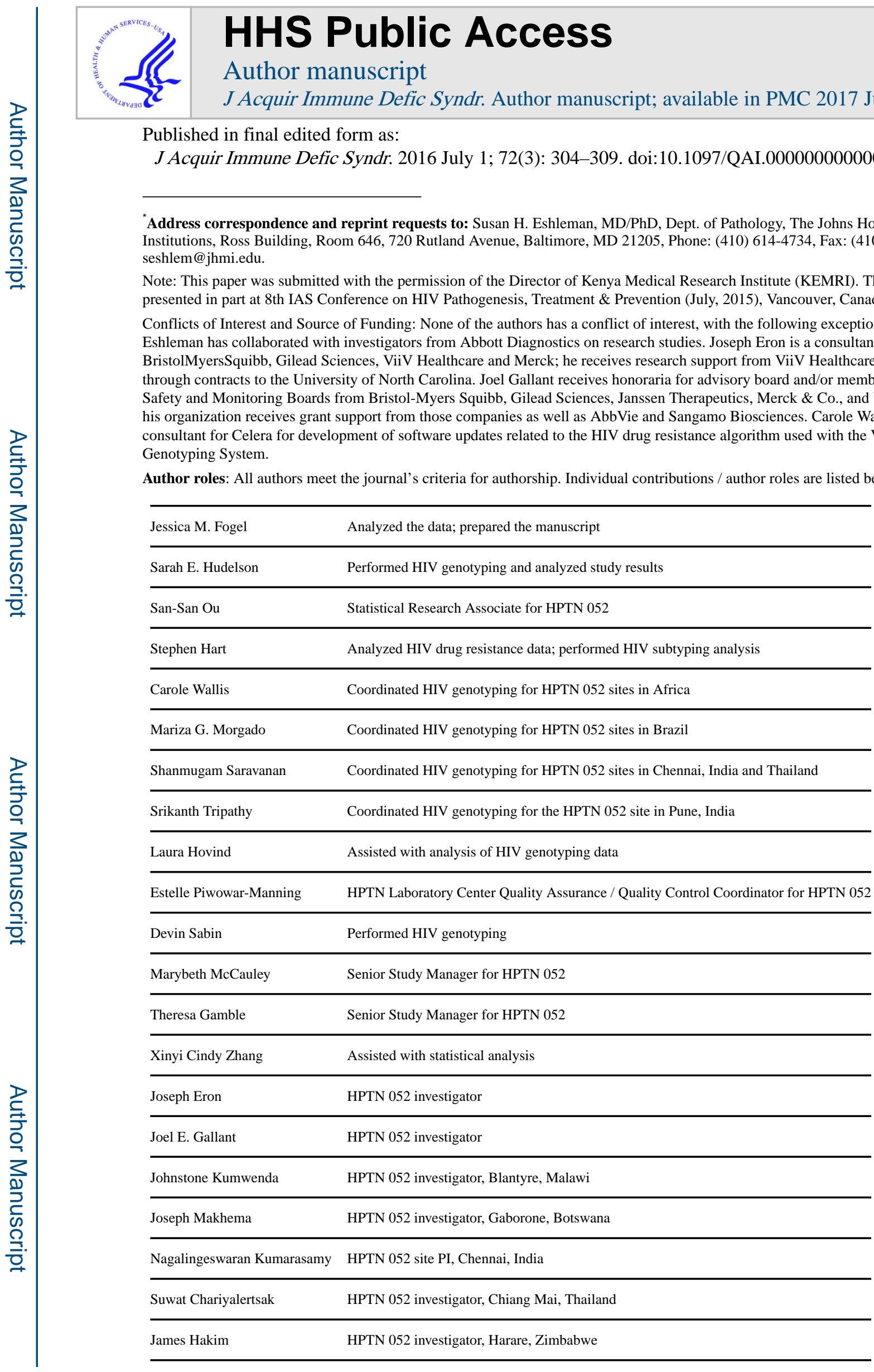




\section{HIV drug resistance in adults failing early antiretroviral treatment: results from the HIV Prevention Trials Network 052 trial}

Jessica M. Fogel, PhD,

Dept. of Pathology, Johns Hopkins Univ. School of Medicine, Baltimore, MD, USA

Sarah E. Hudelson, BS,

Dept. of Pathology, Johns Hopkins Univ. School of Medicine, Baltimore, MD, USA

San-San Ou, MS,

Vaccine and Infectious Disease Division, Fred Hutchinson Cancer Research Center, Seattle, WA, USA

\section{Stephen Hart, PhD,}

Frontier Science \& Technology Research Foundation, Amherst, NY, USA

Carole Wallis, PhD,

Specialty Molecular Division, Lancet Laboratories and BARC-SA, Johannesburg, South Africa

Mariza G. Morgado, PhD,

Laboratory of AIDS and Molecular Immunology, Oswaldo Cruz Institute, Rio de Janeiro, Brazil

Shanmugam Saravanan, PhD,

Y. R. Gaitonade Centre for AIDS Research and Education, Chennai, India

\section{Srikanth Tripathy, MD,}

\begin{tabular}{ll}
\hline Sharlaa Badal-Faesen & HPTN 052 investigator, Johannesburg, South Africa \\
\hline Victor Akelo & HPTN 052 investigator, Kisumu, Kenya \\
\hline Mina Hosseinipour & HPTN 052 investigator, Lilongwe, Malawi \\
\hline Breno Riegel Santos & HPTN 052 investigator, Porto Alegre RS, Brazil \\
\hline Sheela V. Godbole & HPTN 052 investigator, Pune, India \\
\hline Jose Henrique Pilotto & HPTN 052 site PI, HGNI, Rio de Janeiro, Brazil \\
\hline Beatriz Grinsztejn & HPTN 052 site PI, Fiocruz, Rio de Janeiro, Brazil \\
\hline Ravindre Panchia & HPTN 052 site PI, Soweto, South Africa \\
\hline Kenneth H. Mayer & HPTN 052 site PI, Boston, USA \\
\hline Ying Q. Chen & Protocol Statistician for HPTN 052 \\
\hline Myron S. Cohen & Protocol Chair for HPTN 052 \\
\hline Susan H. Eshleman & Virologist for HPTN 052; designed the study; analyzed the data; prepared the manuscript \\
\hline
\end{tabular}


National JALMA Institute for Leprosy and Other Mycobacterial Diseases, Agra, India

Laura Hovind, MS,

Frontier Science \& Technology Research Foundation, Amherst, NY, USA

Estelle Piwowar-Manning, BS MT (ASCP),

Dept. of Pathology, Johns Hopkins Univ. School of Medicine, Baltimore, MD, USA

Devin Sabin, MS,

Dept. of Pathology, Johns Hopkins Univ. School of Medicine, Baltimore, MD, USA

Marybeth McCauley, MPH,

Science Facilitation Department, FHI 360, Washington, DC, USA

Theresa Gamble, PhD,

Science Facilitation Department, FHI 360, Durham, NC, USA

Xinyi Cindy Zhang, PhD,

Vaccine and Infectious Disease Division, Fred Hutchinson Cancer Research Center, Seattle, WA, USA

Joseph J. Eron, MD,

Dept. of Medicine, Univ. of North Carolina at Chapel Hill, Chapel Hill, NC, USA

Joel E. Gallant, MD, MPH,

Southwest CARE Center, Santa Fe, NM, USA

Johnstone Kumwenda, FRCP,

College of Medicine-Johns Hopkins Project, Blantyre, Malawi

Joseph Makhema, MBChB, FRCP,

Botswana Harvard AIDS Institute, Gaborone, Botswana

Nagalingeswaran Kumarasamy, MBBS, PhD,

YRGCARE Medical Centre, VHS, Chennai, India

Suwat Chariyalertsak, MD, Dr.PH,

Research Institute for Health Sciences, Chiang Mai University, Chiang Mai, Thailand

James Hakim, MD,

Dept. of Medicine, Univ. of Zimbabwe, Harare, Zimbabwe

Sharlaa Badal-Faesen, MBBCh,

Univ. of Witwatersrand, Johannesburg, South Africa

Victor Akelo, MBChB, MPH,

Kenya Medical Research Institute, Kisumu, Kenya; Center for Disease Control, Kisumu, Kenya

Mina C. Hosseinipour, MD, MPH,

Division of Infectious Diseases, Univ. of North Carolina at Chapel Hill, Chapel Hill, NC, USA; UNC

Project-Malawi, Institute for Global Health and Infectious Diseases, Lilongwe, Malawi

Breno Riegel Santos, MD,

Serviço de Infectologia, Hospital Nossa Senhora da Conceição, Porto Alegre, Brazil 
Sheela V. Godbole, MD, PGDEPI, National AIDS Research Institute (ICMR), Pune, India

Jose Henrique Pilotto, MD,

Hospital Geral de Nova Iguacu and Laboratorio de AIDS e Imunologia Molecular-IOC/Fiocruz, Rio de Janeiro, Brazil

Beatriz Grinsztejn, MD, PhD,

Instituto Nacional de Infectologia Evandro Chagas-INI-Fiocruz, Rio de Janeiro, Brazil

Ravindre Panchia, MBBCh,

University of the Witwatersrand, Perinatal HIV Research Unit, Soweto HPTN CRS, Soweto, South

Africa

Kenneth H. Mayer, MD,

Fenway Health and Infectious Disease Division, The Fenway Institute; Beth Israel Deaconess

Medical Center/Dept. of Medicine, Harvard Medical School, Boston, MA, USA

Ying Q. Chen, PhD,

Vaccine and Infectious Disease Division and Public Health Sciences Division, Fred Hutchinson

Cancer Research Center, Seattle, WA, USA

Myron S. Cohen, MD, and

Dept. of Medicine, Univ. of North Carolina at Chapel Hill, Chapel Hill, NC, USA

Susan H. Eshleman, MD, PhD

Dept. of Pathology, Johns Hopkins Univ. School of Medicine, Baltimore, MD, USA

\section{Abstract}

Early initiation of antiretroviral therapy (ART) reduces HIV transmission and has health benefits. HIV drug resistance can limit treatment options and compromise use of ART for HIV prevention. We evaluated drug resistance in 85 participants in the HPTN 052 trial who started ART at CD4 counts of 350-550 cells $/ \mathrm{mm}^{3}$ and failed ART by May 2011; 8.2\% had baseline resistance and $35.3 \%$ had resistance at ART failure. High baseline viral load and less education were associated with emergence of resistance at ART failure. Resistance at ART failure was observed in 7/8 (87.5\%) participants who started ART at lower CD4 cell counts.

\section{Keywords}

HIV; HPTN 052; early ART; ART failure; resistance

\section{INTRODUCTION}

The multi-national HIV Prevention Trials Network (HPTN 052) trial showed that early initiation of antiretroviral treatment (ART) significantly reduces sexual HIV transmission in serodiscordant couples. ${ }^{1,2}$ Early ART initiation has also been shown to have health benefits for the HIV-infected individual receiving treatment, including lower rates of severe illness ${ }^{3-5}$ and increased survival. ${ }^{6}$ In the United States (US), ART has been recommended for all HIVinfected individuals regardless of CD4 cell count since 2012. ${ }^{7,8}$ The World Health 
Organization guidelines were recently changed to recommend ART for all HIV-infected individuals, regardless of CD4 cell count. ${ }^{9}$

Use of ART for HIV treatment and prevention can be compromised by HIV drug resistance, especially in resource-limited settings where resistance testing is not routinely performed as part of clinical management. There is relatively little information available about emergence of HIV drug resistance in individuals who initiate ART at higher CD4 cell counts. Observational studies in the United Kingdom and North America have reported a lower prevalence of treatment emergent HIV drug resistance among patients who start ART early. ${ }^{10-12}$ However, little is known about factors associated with drug resistance in HIVinfected individuals who initiate ART at higher CD4 cell counts, or in settings where ART is used for HIV prevention. In this study, we analyzed HIV drug resistance among HIVinfected individuals who failed ART in the HPTN 052 trial before the interim study report was released.

\section{METHODS}

\section{Study cohort}

HPTN 052 (NCT00074581) was a Phase 3, randomized, controlled clinical trial that enrolled HIV serodiscordant couples in Africa, Asia, and the Americas. ${ }^{1-3}$ HIV-infected (index) participants had CD4 cell counts of 350-550 cells $/ \mathrm{mm}^{3}$ at enrollment. In the early ART arm, index participants initiated ART immediately after enrollment. In the delayed ART arm, index participants initiated ART when their CD4 cell count was $<250$ cells $/ \mathrm{mm}^{3}$ on two consecutive study visits, or when they developed an AIDS-defining illness. ${ }^{1}$ Viral load was measured quarterly.

Enrollment criteria for index participants included no prior antiretroviral (ARV) drug use, except for short-term regimens for prevention of mother-to-child transmission. Participants who had a viral load $\$ 400$ copies $/ \mathrm{mL}$ at enrollment were excluded from the analyses; some of those participants were found to be on ART at the time of enrollment, but did not disclose this to study staff. ${ }^{13}$ The most common ART regimen used was a combination of efavirenz, lamivudine, and zidovudine. ${ }^{1}$ This report includes analysis of data from the start of the trial (June 2007) through May 2011 (interim report of the primary study outcome).

\section{Laboratory methods}

HIV viral load and CD4 cell count were determined at study sites. ${ }^{1}$ HIV genotyping was performed retrospectively using the ViroSeq HIV-1 Genotyping System, v2.8 (Celera Diagnostics, Alameda, CA). This testing was performed at four study sites (Pune and Chennai, India; Johannesburg, South Africa; Rio de Janeiro, Brazil) and at the HPTN Laboratory Center (Baltimore, MD, USA). Resistance results were calculated using the Resistance Calculator program at Frontier Science Foundation using the Stanford v6.3 algorithm. ${ }^{14}$ Sequences were submitted to GenBank (accession numbers: KT833391KT833560, KU562071-KU562085). HIV subtyping was performed by phylogenetic analysis. Laboratories that performed HIV genotyping participated in the US Division of 
AIDS Virology Quality Assurance program. ${ }^{15}$ Laboratories that performed CD4 cell count testing participated in the UK NEQAS external quality assurance program. ${ }^{16}$

\section{Statistical analysis}

Viral suppression was defined as the first of two consecutive viral load measurements $\$ 400$ copies $/ \mathrm{mL}$ after ART initiation. ART failure was defined as the first of two consecutive viral load measurements $>1,000$ copies $/ \mathrm{mL}$ after 24 weeks on ART. HIV drug resistance was assessed by testing samples collected at ART initiation (baseline) and ART failure. Demographic and clinical factors were analyzed for association using logistic regression. The association of baseline resistance with time to viral suppression after ART initiation was analyzed using Cox proportional hazards model. Analyses were performed using SAS software, v9.4 (SAS Institute, Cary, NC, USA).

\section{Ethical considerations}

Written informed consent was obtained from all participants in the HPTN 052 trial. The trial was approved by Institutional Review Boards/Ethics Committees at each participating institution.

\section{RESULTS}

\section{Study cohort}

In HPTN 052, 886 index participants were randomized to the early ART arm; 95 (10.7\%) of those participants failed ART by May 2011. Ten participants were excluded from analysis (two had viral loads $\$ 400$ copies $/ \mathrm{mL}$ at enrollment and eight did not have paired genotyping results from baseline and ART failure visits). The remaining 85 participants had a median follow-up of 1.99 years (range: $0.74-5.65$ ) in the period assessed. The median baseline $\log _{10}$ viral load was 4.42 copies/mL (IQR: 3.76-4.86) and the median baseline CD4 cell count was 459.5 cells $/ \mathrm{mm}^{3}$ (IQR: $373-542.5$ ). The majority (39/42=92.9\%) of HIV infections at the African sites were subtype C; other infections included varied HIV subtypes with one to eight infections of each subtype (Table). Therefore, HIV subtype was not included in the analyses below. We did not observe an association between region (Africa, the Americas, Asia) and resistance in any of the analyses described below.

In the delayed ART arm, 213 (24.0\%) of 887 index participants started ART in this study and nine failed ART by May 2011. Genotyping results were obtained for 7/9 participants at baseline and eight of those participants at ART failure (six had paired baseline/failure results).

\section{HIV drug resistance}

In the early ART arm, seven (8.2\%) of the 85 participants who failed ART had drug resistance mutations detected at baseline (including four women, one of whom reported prior use of ARV drugs during pregnancy, Figure). Three had non-nucleoside reverse transcriptase inhibitor (NNRTI) resistance only, one had nucleoside/nucleotide reverse transcriptase inhibitor (NRTI) resistance only, and three had NNRTI and NRTI resistance. Thirty (35.3\%) of the 85 participants had drug resistance mutations detected at ART failure;

J Acquir Immune Defic Syndr. Author manuscript; available in PMC 2017 July 01. 
19 had NNRTI resistance only, three had NRTI resistance only, and eight had NNRTI and NRTI resistance (Figure). Twenty-seven (90.0\%) of the 30 participants had resistance mutations detected at failure that were not present at baseline. Twenty-three had new NNRTI resistance; all had new resistance to efavirenz, and most had cross-resistance to other NNRTIs. Eight had new NRTI resistance; all had new resistance to lamivudine and emtricitabine; one also had new resistance to abacavir and didanosine. Protease inhibitor resistance was not observed in any samples.

In the delayed ART arm, baseline resistance was detected in two (28.6\%) of seven participants with genotyping results. At ART failure, seven (87.5\%) of eight participants who had genotyping results had resistance, including all six participants with paired baseline/failure results. Both of the participants who had baseline resistance acquired new resistance at failure. The frequency of resistance at ART failure was significantly higher in the delayed ART arm compared to the early ART arm (7/8 [87.5\%] vs. 30/85 [35.2\%], $\mathrm{P}=0.006$, Fisher's exact test).

\section{Factors associated with HIV drug resistance}

Because only nine participants in the delayed ART failed ART by May 2011 arm, factors associated with HIV drug resistance were assessed in the early ART arm only. None of the clinical or demographic factors analyzed were associated with baseline drug resistance among those who failed ART (Table). Higher baseline viral load was associated with resistance at ART failure and new resistance at ART failure. Having primary or secondary schooling (compared to no education/schooling) was associated with a lower frequency of resistance at ART failure and new resistance at ART failure. Baseline drug resistance was not associated with time to viral suppression after ART initiation among the 85 participants who failed ART; 50 (58.8\%) of the 85 participants never achieved viral suppression before meeting the criteria for ART failure. There was no difference ( $\mathrm{P}=0.2$, Chi-square test) in the frequency of new resistance at ART failure among those who never achieved viral suppression $(15 / 50=30.0 \%)$, those who achieved viral suppression and remained virally suppressed prior to ART failure $(10 / 28=35.7 \%)$, and those who achieved viral suppression but had intermittent viremia prior to ART failure $(2 / 7=28.6 \%)$.

\section{DISCUSSION}

HIV drug resistance may compromise use of ART for HIV treatment and prevention. ART is now recommended for all HIV-infected individuals, regardless of CD4 cell count. ${ }^{7-9,17,18}$ However, few studies have evaluated HIV drug resistance when ART is initiated at higher CD4 cell counts. Among participants who failed ART in the early ART arm of HPTN 052 (data through May 2011), 8.2\% had resistance at the time of ART initiation. This is similar to the frequency of baseline resistance among participants who failed ART in the ACTG 5175 study (PEARLS), which enrolled participants at many of the same study sites as HPTN 052. In PEARLS, ART was initiated at lower CD4 cell counts $\left(<300\right.$ cells $\left./ \mathrm{mm}^{3}\right)$; the frequency of baseline resistance in PEARLS was $9.4 \%$ among those who failed ART, and $4.3 \%$ among those in a case control group of those who did not fail ART. ${ }^{19}$ 
In HPTN 052, the frequency of resistance at ART failure was significantly lower in the early ART arm than in the delayed ART arm (35.3\% vs. $87.5 \%, \mathrm{P}=0.006)$. It is important to note, however, that very few participants in the delayed ART arm failed ART by May 2011. In other studies where ART was initiated at lower CD4 cell counts, the frequency of resistance at ART failure was also higher than that observed in the early ART arm of HPTN 052 (70$84 \%$ in two clinical cohort studies in sub-Sarahan Africa; ${ }^{20,21} 50 \%$ in a in a randomizedcontrolled clinical trial in the United States ${ }^{22}$ ). Many factors may have contributed to the lower rate of HIV drug resistance observed in the early ART arm of HPTN 052. In HPTN 052, participants in both study arms received adherence counseling and had quarterly viral load monitoring. Participants in the early ART arm may have been more likely to adhere to ART, since they were taking ART primarily as an intervention to prevent partner infection, rather than for their own health.

Among participants in the early ART arm who failed ART, there was a strong association between higher baseline viral load and emergence of resistance at ART failure. This is consistent with results from an observational cohort study in Canada that reported an association of high baseline viral load and resistance at ART failure when ART was initiated at lower CD4 cell counts (median 280 cells $/ \mathrm{mm}^{3}$ ). ${ }^{23}$ Persons with higher baseline viral loads may have more diverse viral populations with a higher frequency of low-level drug resistant variants, or may have higher viral replication rates; these factors could favor emergence of resistant variants at ART failure. Of note, relatively few of the participants analyzed in this study had baseline resistance; therefore, further studies are needed to evaluate the clinical relevance of this association. We also found an association between educational level and resistance at ART failure. Further studies are needed to determine whether this reflects an association between educational level and ART adherence.

More than half of the participants who failed ART in this study never achieved viral suppression. There was no difference in resistance at ART failure among those who never achieved viral suppression, those who achieved viral suppression and remained suppressed until ART failure, and those who achieved viral suppression but had intermittent viremia prior to ART failure. Therefore, emergence of resistance at ART failure in this study does not appear to be driven by selection of resistant variants after ART initiation, before viral suppression was established. This study reports resistance results in participants who failed ART by May 2011, when the interim study report was released (before participants were informed of the benefits of early ART for both the index and partner). Studies are underway to compare drug resistance in the early vs. delayed arms of the trial over the entire trial period and to assess the association of time to viral suppression, ART adherence (assessed through self-report, pill counts, and retrospective ARV drug testing), and drug resistance.

Emergence of HIV drug resistance in the setting of ART is concerning. On-going adherence counseling, frequent viral load monitoring, and prompt cessation of ART at ART failure (with a switch to a new regimen where feasible and appropriate) are likely to maximize the success of ART and reduce the risk of HIV drug resistance in both treatment and prevention settings. 


\section{Acknowledgments}

The authors thank the HPTN 052 study team and participants for providing the samples and data used in this study. We also thank the laboratory staff who helped with sample management and testing.

This work was supported by the grants from the Division of AIDS of the U.S. National Institute of Allergy and Infectious Diseases (NIAID); and by the Office of AIDS Research of the U.S. National Institutes of Health (NIH) [U01-AI068613/UM1-AI068613 (Eshleman); U01-AI068617/UM1-AI068617 (Donnell); and U01-AI068619/ UM1-AI068619 (Vermund/El-Sadr)]. The HPTN 052 trial also received funding through U01-AI018136. Study drugs used in HPTN 052 were donated by Abbott Laboratories, Boehringer-Ingelheim Pharmaceuticals, Inc, Bristol-Myers Squibb, Gilead Sciences, Inc., GlaxoSmithKline/ViiV Healthcare, and Merck \& Co., Inc.

\section{References}

1. Cohen MS, Chen YQ, McCauley M, et al. Prevention of HIV-1 infection with early antiretroviral therapy. N Engl J Med. 2011; 365:493-505. [PubMed: 21767103]

2. Cohen, MS.; Chen, YQ.; McCauley, M., et al. Final results of the HPTN 052 randomized controlled trial: antiretroviral therapy prevents HIV transmission. 8th IAS Conference on HIV Pathogenesis, Treatment \& Prevention; Vancouver, Canada. Jul 2015; Abstract: MOAC0101LB

3. Grinsztejn B, Hosseinipour MC, Ribaudo HJ, et al. Effects of early versus delayed initiation of antiretroviral treatment on clinical outcomes of HIV-1 infection: results from the phase 3 HPTN 052 randomised controlled trial. Lancet Infect Dis. 2014; 14:281-290. [PubMed: 24602844]

4. The TEMPRANO ANRS 12136 Study Group. A trial of early antiretrovirals and isoniazid preventive therapy in Africa. N Engl J Med. 2015; 373:808-822. [PubMed: 26193126]

5. The INSIGHT START Study Group. Initiation of antiretroviral therapy in early asymptomatic HIV infection. N Engl J Med. 2015; 373:795-807. [PubMed: 26192873]

6. Severe P, Juste MA, Ambroise A, et al. Early versus standard antiretroviral therapy for HIV-infected adults in Haiti. N Engl J Med. 2010; 363:257-265. [PubMed: 20647201]

7. Panel on Antiretroviral Guidelines for Adults and Adolescents. Guidelines for the use of antiretroviral agents in HIV-1-infected adults and adolescents. Department of Health and Human Services; Available at: http://www.aidsinfo.nih.gov/ContentFiles/AdultandAdolescentGL.pdf. Accessed: Aug 17, 2015

8. Thompson MA, Aberg JA, Hoy JF, et al. Antiretroviral treatment of adult HIV infection: 2012 recommendations of the International Antiviral Society-USA panel. JAMA. 2012; 308:387-402. [PubMed: 22820792]

9. World Health Organization. Guideline on when to start antiretroviral therapy and on pre-exposure prophylaxis for HIV. 2015. Available at: http://apps.who.int/iris/bitstream/ 10665/186275/1/9789241509565_eng.pdf?ua=1. Accessed: Oct 6, 2015

10. Uy J, Armon C, Buchacz K, et al. Initiation of HAART at higher CD4 cell counts is associated with a lower frequency of antiretroviral drug resistance mutations at virologic failure. $\mathrm{J}$ Acquir Immune Defic Syndr. 2009; 51:450-453. [PubMed: 19474757]

11. Lima VD, Reuter A, Harrigan PR, et al. Initiation of antiretroviral therapy at high CD4+ cell counts is associated with positive treatment outcomes. AIDS. 2015; 29:1871-1882. [PubMed: 26165354]

12. Lodi S, Phillips A, Fidler S, et al. Role of HIV infection duration and CD4 cell level at initiation of combination anti-retroviral therapy on risk of failure. PLoS One. 2013; 8:e75608. [PubMed: 24086588]

13. Fogel JM, Wang L, Parsons TL, et al. Undisclosed antiretroviral drug use in a multi-national clinical trial (HPTN 052). J Infect Dis. 2013; 208:1624-1628. [PubMed: 23908493]

14. Liu TF, Shafer RW. Web resources for HIV type 1 genotypic-resistance test interpretation. Clin Infect Dis. 2006; 42:1608-1618. [PubMed: 16652319]

15. Hollinger FB, Bremer JW, Myers LE, et al. Standardization of sensitive human immunodeficiency virus coculture procedures and establishment of a multicenter quality assurance program for the AIDS Clinical Trials Group. The NIH/NIAID/DAIDS/ACTG Virology Laboratories. J Clin Microbiol. 1992; 30:1787-1794. [PubMed: 1629336] 
16. United Kingdom National External Quality Assessment Service (UK NEQAS). Available at: http:// www.ukneqas.org.uk. Accessed: Sep 12, 2015

17. Hoen B, Bonnet F, Delaugerre C, et al. French 2013 guidelines for antiretroviral therapy of HIV-1 infection in adults. J Int AIDS Soc. 2014; 17:19034. [PubMed: 24942364]

18. World Health Organization. Maximizing the treatment and prevention potential of antiretroviral drugs: early country experiences towards implementing a treat-all policy. 2015. Available at: http:// apps.who.int/iris/bitstream/10665/183599/1/WHO_HIV_2015.35_eng.pdf. Accessed: Sep 12, 2015

19. Kantor R, Smeaton L, Vardhanabhuti S, et al. Pretreatment HIV drug resistance and HIV-1 Subtype $\mathrm{C}$ are independently associated with virologic failure: results from the multinational PEARLS (ACTG A5175) clinical trial. Clin Infect Dis. 2015; 60:1541-1549. [PubMed: 25681380]

20. Hamers RL, Sigaloff KC, Wensing AM, et al. Patterns of HIV-1 drug resistance after first-line antiretroviral therapy (ART) failure in 6 sub-Saharan African countries: implications for secondline ART strategies. Clin Infect Dis. 2012; 54:1660-1669. [PubMed: 22474222]

21. Marconi VC, Sunpath H, Lu Z, et al. Prevalence of HIV-1 drug resistance after failure of a first highly active antiretroviral therapy regimen in KwaZulu Natal, South Africa. Clin Infect Dis. 2008; 46:1589-1597. [PubMed: 18419495]

22. Gulick RM, Ribaudo HJ, Shikuma CM, et al. Three- vs four-drug antiretroviral regimens for the initial treatment of HIV-1 infection: a randomized controlled trial. JAMA. 2006; 296:769-781. [PubMed: 16905783]

23. Harrigan PR, Hogg RS, Dong WW, et al. Predictors of HIV drug-resistance mutations in a large antiretroviral-naive cohort initiating triple antiretroviral therapy. J Infect Dis. 2005; 191:339-347. [PubMed: 15633092] 


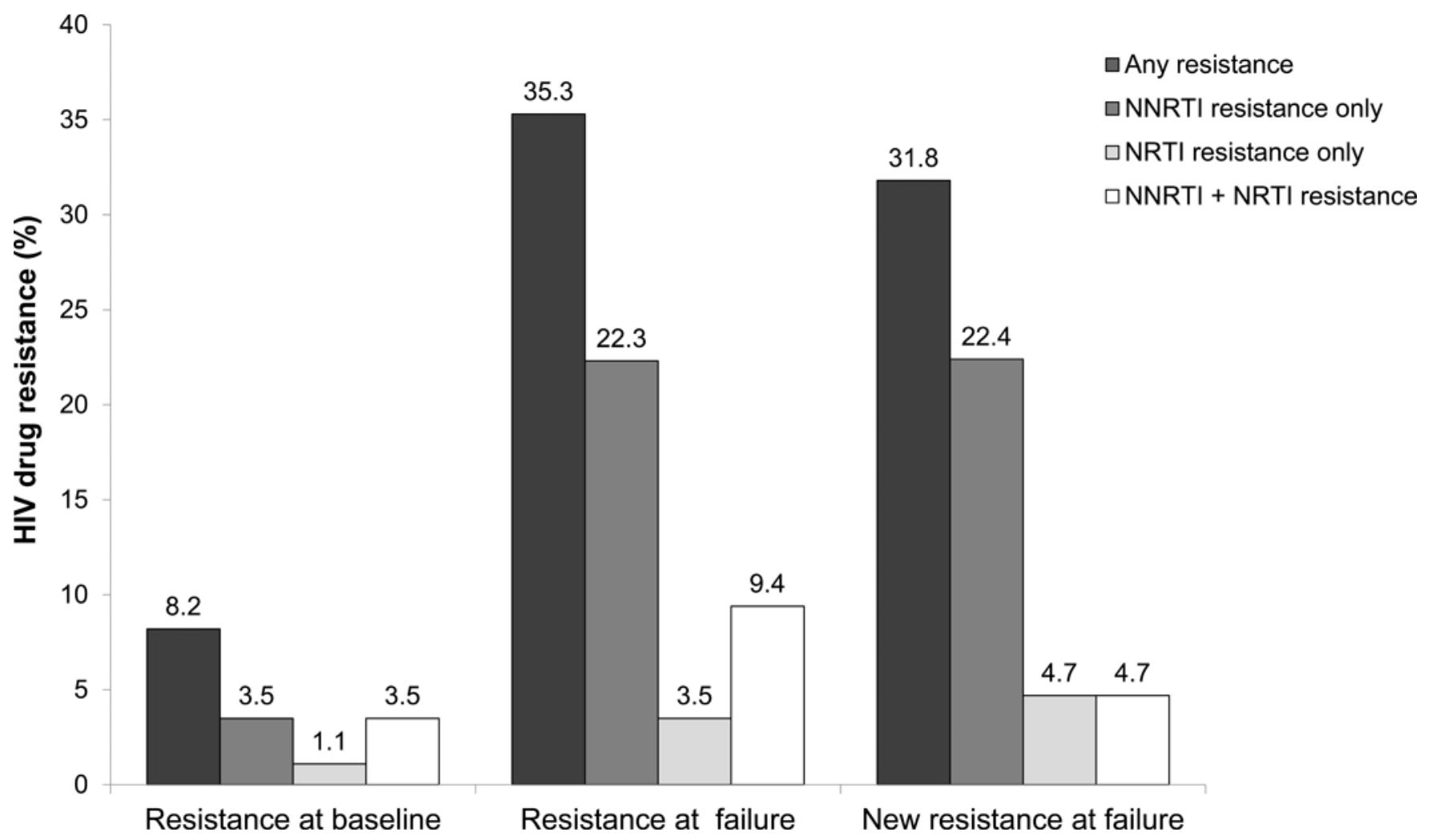

Figure. Proportion of participants with HIV drug resistance at baseline and at treatment failure The figure shows the frequency of HIV drug resistance among participants in the early ART arm of HPTN 052 who failed ART ( $\mathrm{N}=85$ with paired baseline/failure results). Seven (8.2\%) participants had resistance at baseline. The most common NNRTI and NRTI mutations were Y181C and M184V, which were each detected in three of the seven cases. All of the mutations detected at baseline, with the exception of one Y181C mutation, were also present in the corresponding failure samples. Thirty (35.3\%) had resistance at ART failure. The most common NNRTI mutation was K103N, which was detected in $20(66.7 \%)$ of the 30 cases. The most common NRTI mutation was M184V, which was detected in 11 (36.7\%) of the 30 cases. Twenty-seven $(90.0 \%)$ of those 30 participants had one or more resistance mutations detected at failure that was not present at the time of ART initiation (K103N [n=18], M184V [n=8], V106M [n=4), V108I [n=2], K238T [n=2), G190A [n=1), and K101E [n=1]).

Twenty-three $(85.2 \%)$ had new resistance to efavirenz, and most had cross-resistance to other NNRTIs (nevirapine [N=21], rilpivirine [N=2], and etravirine [N=1]). Eight $(29.6 \%)$ had new NRTI resistance; all had new resistance to lamivudine and emtricitabine; one also had new resistance to abacavir and didanosine, due to the acquisition of M184V in addition to the baseline mutations M41L and T215E. Protease inhibitor resistance was not observed in any samples. Among the $78(91.8 \%)$ participants who did not have baseline resistance, 23 $(27.1 \%)$ had resistance at failure (not shown).

Abbreviations: NNRTI: non-nucleoside reverse transcriptase inhibitor; NRTI: nucleoside/ nucleotide reverse transcriptase inhibitor. 


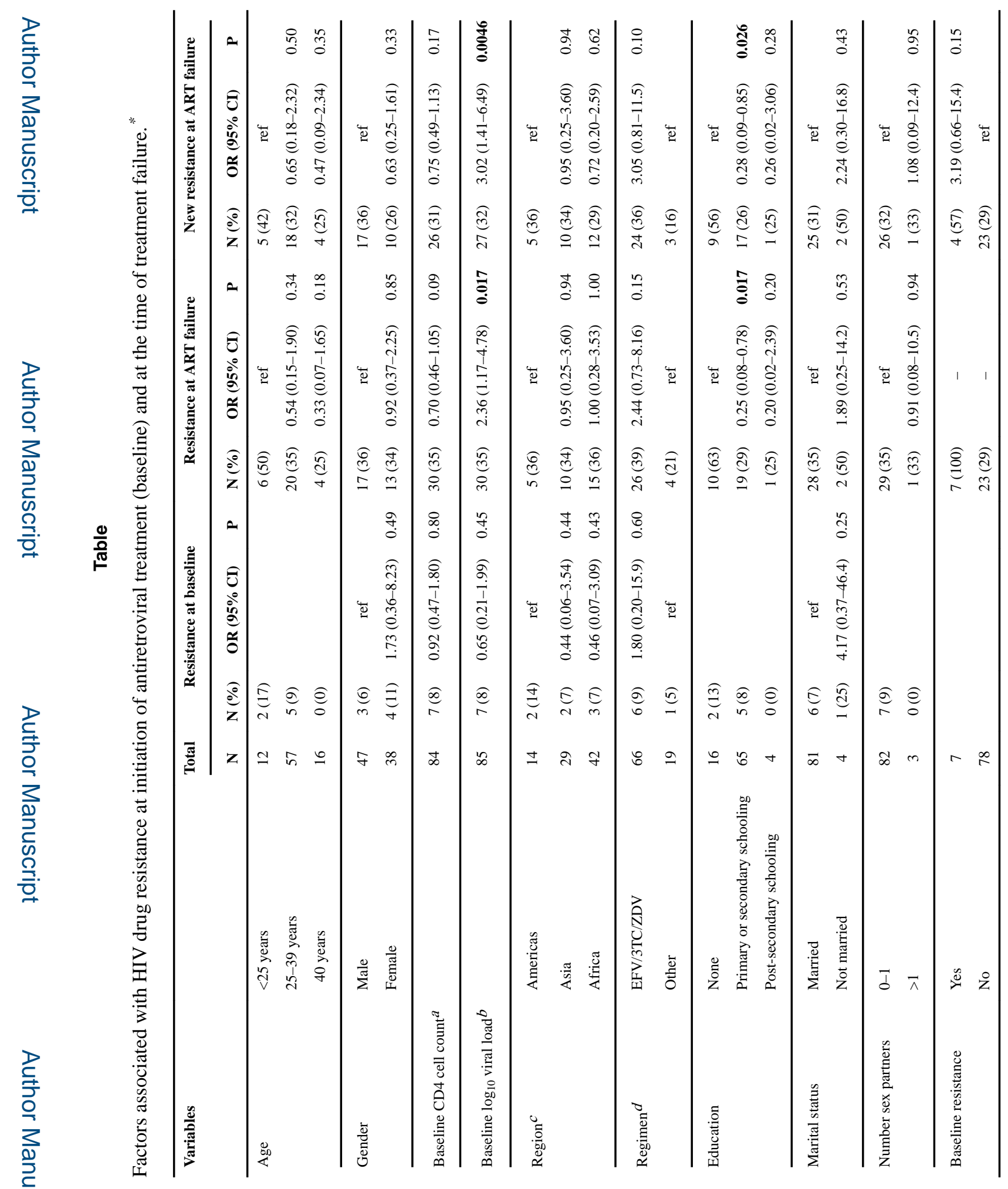

J Acquir Immune Defic Syndr. Author manuscript; available in PMC 2017 July 01. 
Fogel et al.

Page 13

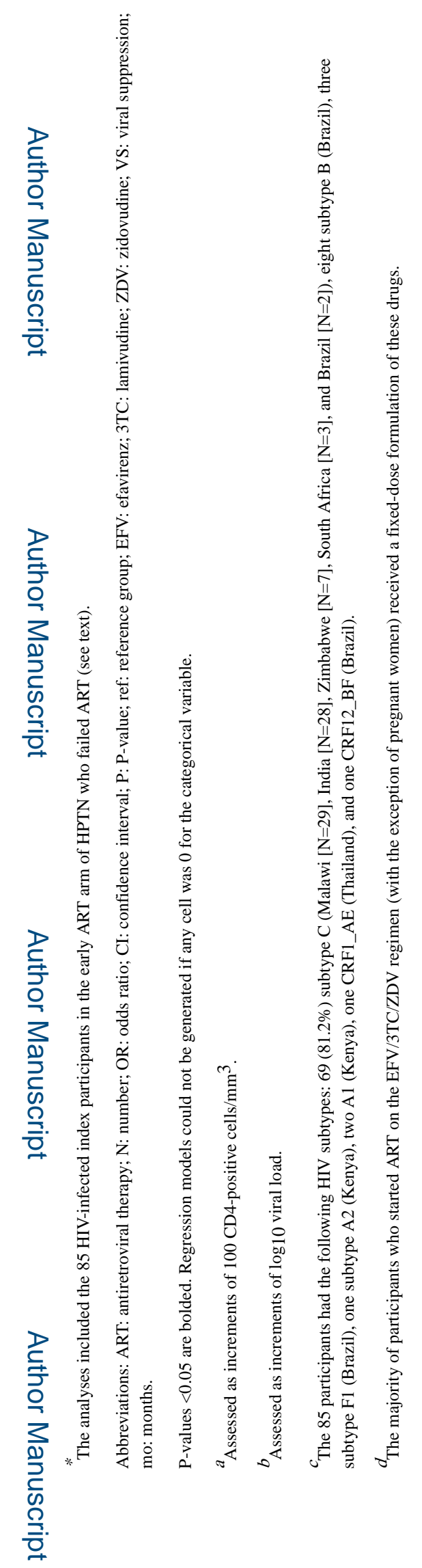

J Acquir Immune Defic Syndr. Author manuscript; available in PMC 2017 July 01. 